\title{
Bifurcations in Van der Pol-Like Systems
}

\author{
Orhan Ozgur Aybar, ${ }^{1,2}$ Ilknur Kusbeyzi Aybar, ${ }^{3}$ and Avadis Simon Hacinliyan ${ }^{2,4,5}$ \\ ${ }^{1}$ Department of Mathematics, Gebze Institute of Technology, Kocaeli, 41400 Gebze, Turkey \\ ${ }^{2}$ Department of Information Systems and Technologies, Yeditepe University, Atasehir, 34755 Istanbul, Turkey \\ ${ }^{3}$ Department of Computer Education and Instructional Technology, Yeditepe University, Atasehir, 34755 Istanbul, Turkey \\ ${ }^{4}$ Department of Physics, Yeditepe University, Atasehir, 34755 Istanbul, Turkey \\ ${ }^{5}$ Department of Physics, Bogazici University, Bebek, 34342 Istanbul, Turkey
}

Correspondence should be addressed to Orhan Ozgur Aybar; oaybar@yeditepe.edu.tr

Received 2 October 2013; Accepted 25 November 2013

Academic Editor: Panos Liatsis

Copyright (C) 2013 Orhan Ozgur Aybar et al. This is an open access article distributed under the Creative Commons Attribution License, which permits unrestricted use, distribution, and reproduction in any medium, provided the original work is properly cited.

\begin{abstract}
Generalizations of the Van der Pol system with polynomial interactions involving additional parameters are studied in order to understand qualitative properties such as stability and additional bifurcations in these generalized Van der Pol systems. The generalizations include the one known as the Duffing-Van der Pol system which has properties similar to those of the MaxwellBloch system.
\end{abstract}

\section{Introduction}

Dynamical systems underlying many oscillatory real life problems that arise in applied sciences can be considered as generalizations of the Van der Pol (henceforth referred to as VP) system [1]. Due to its wide area of applications in a number of scientific areas such as electronics and geology, the VP system stands as an important example of dynamical systems. It was first developed by the Dutch physicist Balthasar Van der Pol (1889-1959) to model power amplifiers in vacuum tube radios exhibiting negative resistance [1]. Methods referred to by names such as averaging by Guckenheimer and Holmes [2] and two-variable expansion by Rand and Armbruster [3] have been applied to this system. Related types of averaging methods have also been applied by Strogatz [4], Verhulst [5, 6], Sanders et al. [7], Kuznetsov [8], Jordan and Smith [9], and Hale and Koçak [10] to similar oscillatory problems. We use standard averaging in the rest of this paper $[2,5,7]$. Oscillatory solutions to dynamical systems using the center manifold and normal form methods, and bifurcation and stability analysis have been extensively studied by Cao and Xiao [11-13]. This paper aims to look at possible generalizations of VP and Duffing-like systems and to study their bifurcation schemes in order to clarify our understanding. The methodology, some of the observed bifurcation scenarios, and attractor structures resemble those reported by Cao and Xiao in other systems [11-13].

Section 2 discusses quadratic generalizations of the VP system including the one known as the Duffing-Van der Pol (henceforth referred to as DVP) system and compares the averaging results with the averaged versions of some commonly known similar dynamical systems. Section 3 introduces a new generalization for the DVP system and investigates results obtained by methods discussed in previous sections. In Section 4, generalizations that introduce higher nonlinearities are studied in the same manner. Overall results are compared and discussed in Section 5.

The VP equation is the second-order ordinary differential equation

$$
\ddot{x}-\mu\left(1-x^{2}\right) \dot{x}+x=0
$$

modeling the VP oscillator [1]. The part of the VP equation including $\dot{x}$ together with its coefficient implies that the friction or the resistance represented by the nonlinear-damping term changes sign and becomes negative as the amplitude 
increases. This model can be derived from the Rayleigh equation [14] and can be reduced to simple harmonic motion for $\mu=0$ with the solution $y(t)=c_{1} \cos (t)+c_{2} \sin (t)\left(c_{1}, c_{2} \neq 0\right)$.

The generalized models under study can be stated as vector equations given in system (2) by the transformation $(x, \dot{x}) \rightarrow(x, y)$ in order to use dynamical systems approaches for their study $[5,12]$.

The generalized VP equations [1] under study are special cases of the following system:

$$
\begin{gathered}
\dot{x}=a y, \\
\dot{y}=P(x)+Q(x) y,
\end{gathered}
$$

where $P(x)$ and $Q(x)$ are polynomials in the variable $x$ and we can always set $a=1$ by $\bar{y} \rightarrow$ ay and $\bar{P}(x) \rightarrow a P(x)$. System (2) is also known as the Liénard system [15].

Additional parameters in the nonlinearities can be introduced to generate and analyze further bifurcation varieties as follows:

$$
\begin{gathered}
\dot{x}=y, \\
\dot{y}=-\alpha x+\beta y\left(1-x^{2}\right),
\end{gathered}
$$

so that the generalized VP equation is now

$$
\ddot{x}+\alpha x-\beta\left(1-x^{2}\right) \dot{x}=0 .
$$

We carry out the stability analysis by looking into the eigenvalues of the linearized matrix of coefficients. In this manner, the system has a trivial equilibrium point at the origin with the eigenvalues $\left\{(1 / 2)\left(\beta \pm \sqrt{\beta^{2}-4 \alpha}\right)\right\}$. Scaling the time by the undamped angular frequency $\sqrt{\alpha}$ reveals that the only nontrivial parameter is $\beta \sqrt{(1 / \alpha)}(\alpha \neq 0)$, so that $\beta$ itself affects directly the stability of the system.

Bifurcation analysis is the key to understanding the qualitative behavior of a dynamical system. Qualitative changes, if any, in the system when any of the parameters are varied, represent bifurcations and help us understand the behavior of the system better. Using the software package MATCONT, bifurcations of the systems of concern are studied numerically [8]. Choosing $\beta$ as the bifurcation parameter, the obvious scenario for the VP system is obtained such as a family of limit cycles growing endlessly from the Hopf bifurcation point at the trivial equilibrium point indicated by a nonnegative first Lyapunov coefficient [2]. Considering that in a physical system all parameters are nonnegative, it can be said that the equilibrium point of system (3) is always unstable. Details of other bifurcation properties for the simple VP system as given in system (3) including transcritical bifurcation are omitted here for brevity $[9,10,16]$.

Before rescaling, the equation has two nontrivial coefficients, one determining the undamped frequency and the other determining the damping term. The latter changes sign as the amplitude is increased. A generalization of the VP equation should involve either or both of these terms. Our object of study will be generalizations where nonlinear functions on $x$ will be used instead of the term $-\alpha x$ in $\dot{y}$ equation. Nonlinear functions depending on $x$ can be used as generalizations [17].

\section{The Generalized Van der Pol System including Quadratic Self-Nonlinearity}

Replacing the linear term in the $\dot{y}$ equation of system (3) by the quadratic combination $x(1-x)$ modifies the system to

$$
\begin{gathered}
\dot{x}=y, \\
\dot{y}=-\alpha x(1-x)+\beta y\left(1-x^{2}\right) .
\end{gathered}
$$

In addition to the trivial equilibrium point at the origin that always occurs, a second equilibrium point at $(1,0)$ is observed in this case. The eigenvalues (i.e., the eigenvalues of the coefficients matrix of the linearized system) for the trivial equilibrium point are the same as those of the original one $\left\{(1 / 2)\left(\beta \pm \sqrt{\beta^{2}-4 \alpha}\right)\right\}$ and the eigenvalues for the second equilibrium point are $\{ \pm \sqrt{\alpha}\}$. For $\beta^{2}<4 \alpha$, the origin has pure imaginary eigenvalues that indicate Hopf bifurcation. We want to analyze Hopf bifurcation in this system by using the following theorem.

Theorem 1 (Hopf bifurcation, [8]). A Hopf bifurcation occurs if a planar system $\dot{x}=f(x, \eta), x \in \mathbb{R}^{2}, \eta \in \mathbb{R}$, has the following eigenvalues at origin for sufficiently small $\eta$ taken as bifurcation parameter:

$$
\lambda_{1,2}(\eta)=\xi(\eta) \pm i \gamma(\eta)
$$

where real part $\xi(\eta)$ of $\lambda_{1,2}(\eta)$ is zero for $\eta=0$ and imaginary part $\gamma(\eta)$ of $\lambda_{1,2}(\eta)$ is $\gamma_{0} \neq 0$.

\section{(H.1) The first Lyapunov coefficient defined as}

$$
l_{1}\left(\left.\eta\right|_{\eta=0}\right)=\frac{1}{2 w_{0}^{2}}\left(i g_{20} g_{11}+w_{0} g_{21}\right)
$$

is not equal to zero.

(H.2) Consider $d \xi(\eta) /\left.d \eta\right|_{\eta=0} \neq 0$ (transversality condition).

By using Theorem 1, we require that the determinant of the Jacobian matrix be greater than zero and trace of the Jacobian matrix be zero at the related equilibrium point. According to criterion (H.2) in Theorem 1, Hopf point also obeys the transversality condition if the rate of change of real part of eigenvalues at the related equilibrium (codimensionone Hopf) point is greater than zero which means that eigenvalues of the linearized flow cross the imaginary axis with nonzero derivative when bifurcation parameter $\eta$ is zero $[8,9]$. We use the first Lyapunov coefficient to express Hopf bifurcation criteria for bifurcation parameter $\eta$. When the system has pure imaginary eigenvalues at the related equilibrium (Hopf) point, $l_{1}(\eta) \neq 0$. In the neighborhood of the Hopf point, if the first Lyapunov coefficient $l_{1}(\eta)$ is less than zero, supercritical Hopf bifurcation can be observed; if $l_{1}(\eta)$ is greater than zero, subcritical Hopf bifurcation can be observed $[8,9]$. When these conditions are satisfied, the system has codimension one Hopf bifurcation [8]. If $l_{1}(\eta)$ vanishes and becomes zero, this type is called degenerate (generalized) Hopf Bifurcation [8]. 
We see that trace and determinant of the Jacobian matrix at origin are $\beta$ and $\alpha$, respectively. By applying the conditions, we obtain pure imaginary roots $\pm i \sqrt{\alpha}$ depending on $\beta=0$, $\alpha>0$; the transversality condition gives $\left.(d / d \beta)(\beta / 2)\right|_{\beta=0}=$ $1 / 2>0$ at the origin. Under the assumption $\alpha>0$ and sufficiently small bifurcation parameter $\beta$ [18], we have the following conditions at the origin: if $\alpha>0, \beta>0$, subcritical Hopf bifurcation is observed with negative first Lyapunov coefficient and if $\alpha>0, \beta<0$, supercritical Hopf bifurcation is observed with positive first Lyapunov coefficient [8]. The famous limit cycles of the VP equation are observed to bifurcate from the Hopf bifurcation point (indicated by $\mathrm{H}$ ) of the system (5) as shown in Figure 1. When $\beta=0$, the first Lyapunov coefficient is zero.

The determinant and trace at the other equilibrium point $(1,0)$ are $-\alpha$ and zero, respectively. By using the above conditions, $\alpha$ is less than zero and rate of change in real part of eigenvalues which is expected to be nonzero vanishes. At this equilibrium point we thus do not observe Hopf bifurcation as shown in Figure 1 according to Theorem 1.

Definition 2 (normal form expansion). Let the system of differential equation $\dot{x}=f(x, y)$ and $\dot{y}=g(x, y)$ be given where $x, y \in R$ and $f, g \in R \rightarrow R$. To consider the system near its equilibrium point $x=x_{0}$ and $y=y_{0}$ such that $f\left(x_{0}, y_{0}\right)=0$ and $g\left(x_{0}, y_{0}\right)=0$, this point is moved to the origin by $x=x-x_{0}$ and $y=y-y_{0}$. Taylor expansion near $x=0$ and $y=0$ gives

$$
\begin{aligned}
& \dot{x}=a_{11} x+b_{12} y+f_{2}(x, y)+\cdots, \\
& \dot{y}=a_{21} y+b_{22} y+g_{2}(x, y)+\cdots,
\end{aligned}
$$

where $a_{11}, a_{21}, b_{12}, b_{22}$ are coefficients and $f_{2}(x, y)$ and $g_{2}(x, y)$ contain terms of degree two and higher. Diagonalizing or bringing the linear part to the Jordan canonical form by a linear transformation produces

$$
\left(\begin{array}{c}
\dot{x} \\
\dot{y}
\end{array}\right)=\left(\begin{array}{ll}
J_{11} & J_{12} \\
J_{21} & J_{22}
\end{array}\right)\left(\begin{array}{l}
x \\
y
\end{array}\right)+\left(\begin{array}{l}
\widetilde{f}_{2}(x, y) \\
\widetilde{g}_{2}(x, y)
\end{array}\right)
$$

where $J_{i j}, i, j \in\{1,2\}$ denote Jordan canonical form terms of the linear part in system (8). Then a sequence of near identity transformations of the form

$$
\begin{aligned}
& x=\nu+F_{2}(\nu, \vartheta)+\cdots, \\
& y=\vartheta+G_{2}(\nu, \vartheta)+\cdots
\end{aligned}
$$

is applied to simplify the system $(8)$, where $F_{2}(\nu, 9)$ and $G_{2}(\nu, \vartheta)$ contain terms of degree two and higher. The normal form expansion is given as follows:

$$
\begin{aligned}
& \dot{v}=a_{11} \nu+b_{12} \vartheta+\widetilde{F}_{2}(\nu, \vartheta)+\cdots, \\
& \dot{\vartheta}=a_{21} \nu+b_{22} \vartheta+\widetilde{G}_{2}(\nu, \vartheta)+\cdots,
\end{aligned}
$$

where $\widetilde{F}_{2}(\nu, \vartheta)$ and $\widetilde{G}_{2}(\nu, \vartheta)$ contain degree two and higher order terms.

We use normal form expansion around the trivial equilibrium point (origin) to yield Hopf bifurcation as defined

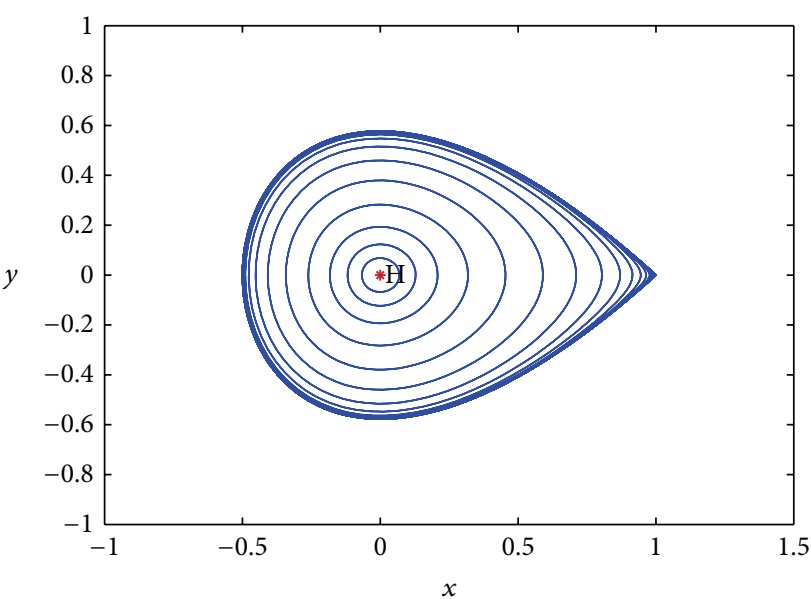

FIgUre 1: Phase planes of system (5) with $\alpha=1, \beta$ variable. The degenerate Hopf bifurcation point (indicated by $\mathrm{H}$ ) is observed at the trivial equilibrium point $(0,0)$.

in Definition 2. In the rest of this work, $v$ and $\vartheta$ will refer to the variables in the near identity transformation [19]. For example, system (5) has the following normal form expansion up to the fifth order which leads to degenerate Hopf bifurcation at origin for $\beta=0$

$$
\begin{gathered}
\dot{\nu}=\frac{i \nu}{\alpha^{3 / 2}}\left(-\alpha^{2}+\frac{5 \alpha}{3}(\nu \vartheta)+\frac{785}{108}(\nu \vartheta)^{2}\right), \\
\dot{\vartheta}=\frac{i \vartheta}{\alpha^{3 / 2}}\left(\alpha^{2}-\frac{5 \alpha}{3}(\nu \vartheta)-\frac{785}{108}(\nu \vartheta)^{2}\right),
\end{gathered}
$$

which is also consistent with the Hopf bifurcation observed at origin in Figure 1.

Figure 2 gives the bifurcation curves with lines indicating pitchfork bifurcation points (indicated by BP). The bifurcation analysis while relaxing the bifurcation parameter $\alpha$ shows a pitchfork and a degenerate limit point bifurcation (indicated by LP) point with limit point coefficient equal to zero as shown in Figure 2. For $\alpha=0, y=0$, the $x$ axis contains equilibrium points with corresponding eigenvalues $\lambda_{1,2}=$ $\{0, \beta\}$ that satisfy the limit point (saddle-node) bifurcation criteria $[5,8]$.

\subsection{Averaging of the Generalized Van der Pol System including} Quadratic Self-Nonlinearity. The method of averaging is a powerful perturbation method which gives a normal form that can be compared to more commonly known dynamical systems. Numerically obtained results concerning stability and bifurcation can also be analytically confirmed using the method of averaging. In order to understand the behavior of the system, we investigate the averaged version of system (5) and observe its similarity to averaged versions of some better known systems.

The averaged equations of system (3) and (5) are calculated by the usual method discussed in $[2,5,7]$. We introduce a small parameter $\epsilon, 0<\epsilon \ll 1$ and replace $\alpha$ and $\beta$ by $1+\epsilon \alpha$, $\epsilon \beta$, respectively, to analyze this system for small parameter 


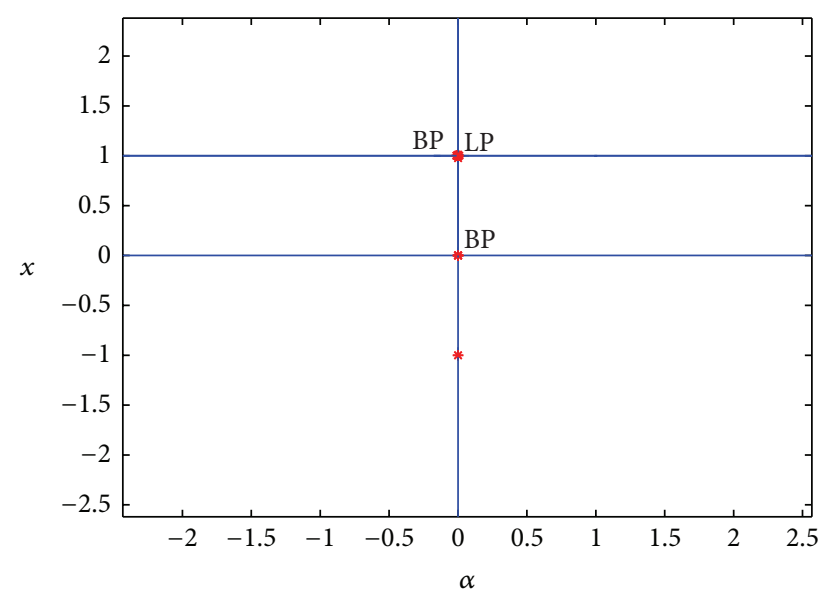

FIGURE 2: Bifurcation graph of system (5) with $\alpha$ variable where a pitchfork bifurcation point (indicated by BP) is observed for $\alpha=0$ that forks a new curve with a limit point bifurcation point (indicated by LP).

values while averaging is carried out over a period in $t$. We use

$$
\begin{aligned}
& x(t)=u(t) \cos t+v(t) \sin t, \\
& y(t)=-u(t) \sin t+v(t) \cos t
\end{aligned}
$$

to obtain

$$
\begin{aligned}
& \dot{u}(t)=\frac{\epsilon}{2}\left(\beta u(t)+\alpha v(t)-\frac{\beta u(t)}{4}\left(u(t)^{2}+v(t)^{2}\right)\right)+O\left(\epsilon^{2}\right), \\
& \dot{v}(t)=\frac{\epsilon}{2}\left(-\alpha u(t)+\beta v(t)-\frac{\beta v(t)}{4}\left(u(t)^{2}+v(t)^{2}\right)\right)+O\left(\epsilon^{2}\right) .
\end{aligned}
$$

According to the theorem given by Verhulst $[5,6]$, the origin is expected to be the only equilibrium point. We calculate $u \dot{u}+v \dot{v}$ and $u \dot{v}-v \dot{u}$ and introduce $r=\sqrt{u^{2}+v^{2}}$ and $\theta=$ $\arctan (v / u)$ to get

$$
\begin{gathered}
\dot{r}=\frac{\epsilon \beta}{2} r\left(1-\frac{r^{2}}{4}\right), \\
\dot{\theta}=\frac{-\epsilon \alpha}{2}
\end{gathered}
$$

in the standard averaging sense $[2,5,7]$. This result shows a typical Hopf bifurcation form $[4,7,8]$. The steady state of $\dot{r}=0$ leads either to the trivial case $r=0$ or to the limit cycle condition $(\beta / 2)\left(1-\left(r^{2} / 4\right)\right)=0$ where $\beta=0$ or $r^{2}=4>0$ implying the existence of a limit cycle of radius 2 . Numerical and averaged solutions are given in Figure 3 that also gives unstable (stable) equilibrium point for $\beta>0(\beta<0)$. For small $\epsilon$, as $\alpha$ is increased, the averaged solution does not tend to the numerical solution of the original equation as shown in Figure 4 . The determinant of the Jacobian matrix at the related equilibrium point $\left(\epsilon^{2} \beta^{2} / 4\right)+\left((\epsilon \alpha-1)^{2} / 4\right)$ is greater than zero for all $\beta, \alpha, \epsilon \in \mathbb{R}$. The trace becomes zero if $\beta$

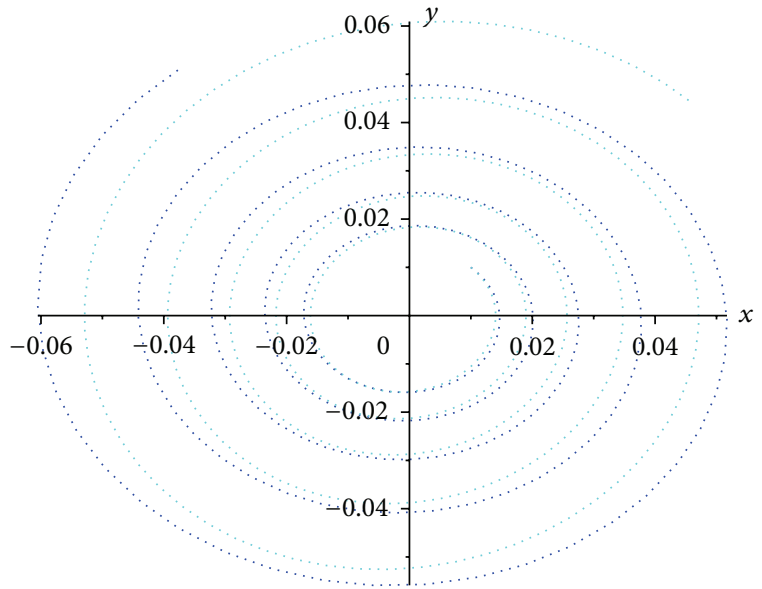

Averaged solutions Numerical solutions

FIGURE 3: Numerical and averaged solutions of system (5) when $\alpha=1, \beta=1$ and $\epsilon=0.1$ near the origin where Hopf bifurcation is observed.

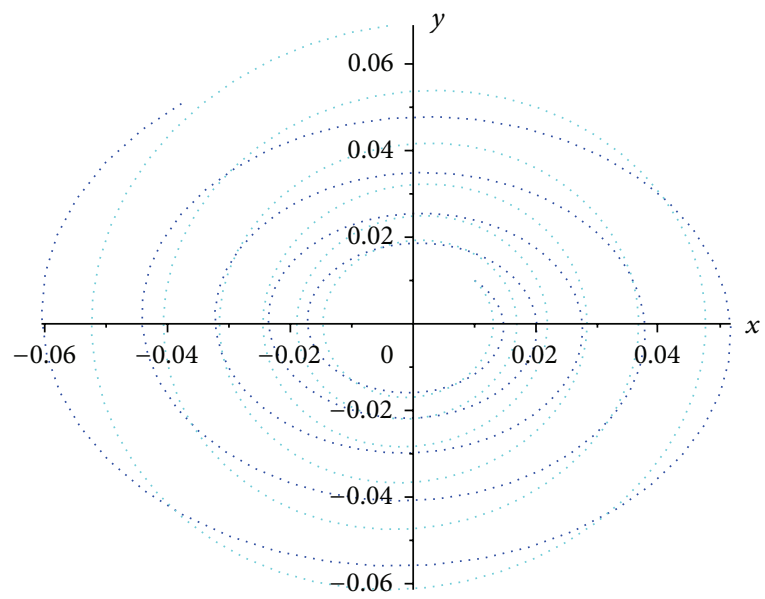

..... Averaged solutions

Numerical solutions

FIGURE 4: Numerical and averaged solutions of system (5) when $\alpha=$ $3, \beta=1$, and $\epsilon=0.1$ near the origin where Hopf bifurcation is observed.

is zero. With the aid of Theorem 1, subcritical (supercritical) case is observed for sufficiently small $\beta>0(\beta<0), 0<$ $\epsilon \ll 1, \alpha>0$ with the first Lyapunov coefficient being less (greater) than zero.

System (5) is a special case of the following averaged version of the general form of generalized VP systems:

$$
\begin{gathered}
\dot{x}=y, \\
\dot{y}=-\alpha x\left(\alpha_{0}+\alpha_{1} x+\alpha_{2} x^{2}\right)+\beta y\left(\beta_{0}+\beta_{2} x^{2}+\beta_{4} x^{4}\right),
\end{gathered}
$$


where we introduce parameters $\alpha_{i}, \beta_{j} \in \mathbb{R},\{i=0,1,2\}$ and $\{j=0,2,4\}$ as coefficients of the generalization terms in parentheses. The averaged form of system (16) calculated by the usual method discussed in $[5,7]$ becomes

$$
\begin{gathered}
\dot{r}=\frac{\epsilon \beta}{2} r\left(\beta_{0}+\frac{\beta_{1}}{4} r^{2}+\frac{\beta_{2}}{8} r^{4}\right), \\
\dot{\theta}=\frac{1-\alpha_{0}(1+\epsilon \alpha)}{2}-\frac{3}{8} \alpha_{2} r^{2}(1-\epsilon \alpha)
\end{gathered}
$$

by introducing $\epsilon, 0<\epsilon \ll 1$ and by replacing $\alpha, \beta$ by $1+\epsilon \alpha, \epsilon \beta$ in that given order.

A number of other systems with linear periodic parts also give similar results upon averaging. For example, the cubic generalization of the Lotka-Volterra system in [19]

$$
\begin{aligned}
& \dot{x}=a x\left(1-x^{2}\right)-b x^{k} y, \\
& \dot{y}=-c y\left(1+y^{2}\right)+d x y^{k}
\end{aligned}
$$

upon averaging (replacing $a, b, c, d$ by $\epsilon a, \epsilon b, \epsilon c, \epsilon d$ ) by the usual method discussed in $[5,7]$ gives

$$
\dot{r}=\epsilon r\left(\frac{a-c}{2}-\frac{3}{8}(a+c) r^{2}\right),
$$

where $\dot{\theta}=1$ for $k=1$ values and $\dot{\theta}=1+\left(\epsilon r^{2} / 8\right)(b+d)$ for $k=2$.

\section{The Duffing-Van der Pol System}

A further generalization of the VP system that introduces the cubic property of the Duffing system in the potential function is the DVP system given by

$$
\begin{gathered}
\dot{x}=y, \\
\dot{y}=-\alpha x\left(\alpha_{0}+\alpha_{2} x^{2}\right)+\beta y\left(1-x^{2}\right),
\end{gathered}
$$

where parameters $\alpha_{0}$ and $\alpha_{2}$ are introduced in the potential function [14]. Depending on the sign of the parameter $\alpha_{0}$, single or double well potential cases exist. There are three equilibrium points, that is, the trivial equilibrium point at the origin and two equilibrium points at $\left( \pm i \sqrt{\alpha_{0} / \alpha_{2}}, 0\right)$. The eigenvalues at the equilibrium points are

$$
\begin{gathered}
\left\{\frac{1}{2}\left(\beta \pm \sqrt{\beta^{2}-4 \alpha \alpha_{0}}\right)\right\} \\
\left\{\frac{1}{2 \alpha_{2}}\left(\beta\left(\alpha_{0}+\alpha_{2}\right) \pm \sqrt{8 \alpha \alpha_{0} \alpha_{2}^{2}+\beta^{2}\left(\alpha_{0}+\alpha_{2}\right)^{2}}\right)\right\}
\end{gathered}
$$

respectively. When a sufficiently small $\beta$ is chosen as bifurcation parameter, trace of the Jacobian $\beta$ is zero and determinant of the Jacobian matrix is $\alpha \alpha_{0}>0$ at the origin. By using Theorem 1, eigenvalues at the origin are $\left\{ \pm i \sqrt{\alpha \alpha_{0}}\right\}$ that give Hopf bifurcation at the origin as shown in Figure 5. For $\beta>0$ and $\alpha=\alpha_{0}=\alpha_{2}>0$, the first Lyapunov coefficient is less than zero that gives supercritical Hopf bifurcation and, for $\beta<0$ and $\alpha=\alpha_{0}=\alpha_{2}>0$, the first Lyapunov coefficient is greater than zero that gives subcritical Hopf bifurcation.

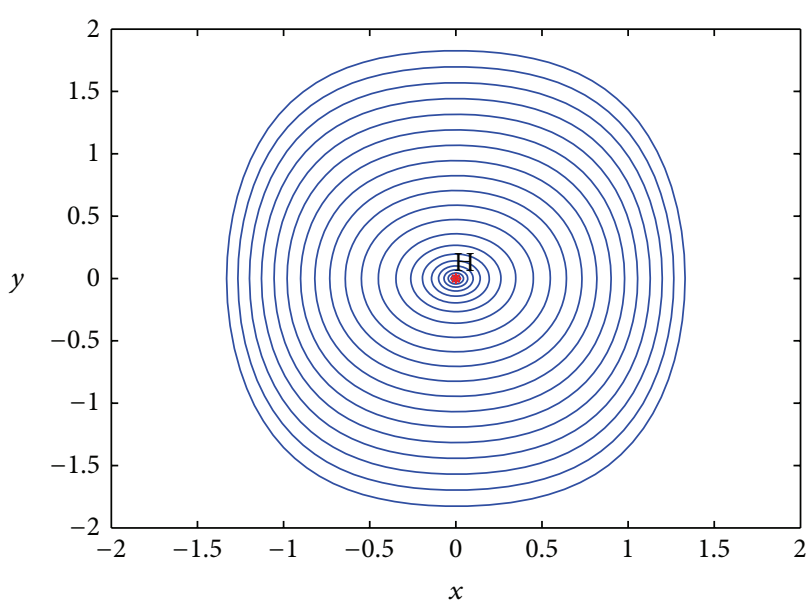

Figure 5: Phase plane of the DVP system (20) when $\alpha=\alpha_{0}=\alpha_{2}=1$ and $\beta$ is varied where Hopf bifurcation is observed at the origin for $\beta=0$.

The Jacobian matrix of system $(20)$ at $\left( \pm i \sqrt{\alpha_{0} / \alpha_{2}}, 0\right)$ is

$$
\left(\begin{array}{cc}
0 & 1 \\
2 \alpha \alpha_{0} & \beta\left(1+\frac{\alpha_{0}}{\alpha_{2}}\right)
\end{array}\right),
$$

where eigenvalues of the Jacobian matrix are purely imaginary if the determinant of the Jacobian matrix is $-2 \alpha \alpha_{0}>0$ and the trace of the Jacobian matrix is $\beta\left(1+\left(\alpha_{0} / \alpha_{2}\right)\right)=0$. These two conditions are used to show Hopf bifurcation. We see that the trace becomes zero when $\beta=0$ or $\alpha_{0} / \alpha_{2}=-1$. By assuming the latter one, the second and third equilibrium points become $( \pm 1,0)$. Another necessary condition is that the rate of change of real part of eigenvalues with respect to bifurcation parameter should be greater than zero where $\beta / 2 \alpha_{2}>0$. Combining these results, we see that Hopf bifurcation occurs if $\beta>0, \alpha>0, \alpha_{2}>0, \alpha_{0}<0$ or $\beta<0, \alpha<0, \alpha_{2}<0, \alpha_{0}>0$ given in Figures 6 and 7 for the bifurcation parameter chosen as $\alpha_{0}$.

As an example, we set the determinant of the Jacobian matrix at the origin to 1 and obtain $\alpha_{0}=1 / \alpha$. Since the pair of eigenvalues cannot have real part according to the Hopf bifurcation condition, we can assume that $\beta=0$ where $\beta \in(-2,2)$ gives pure imaginary eigenvalues $\{ \pm i\}$ as shown in Figure 5. The second and third equilibrium points do not give pure imaginary eigenvalues; hence, we do not observe Hopf bifurcation at these equilibrium points. When $\alpha_{0}=-1 / \alpha$, the second and third equilibrium points possess positive determinants and by using $1+\left(\alpha_{0} / \alpha_{2}\right)=0$ and $\beta=0$, system has pure imaginary eigenvalues $\{ \pm i \sqrt{2}\}$ as shown in Figures 6 and 7. This behavior observed for the single well potential case of the DVP system with parameter $\beta$ free is similar to that of the Lorenz system in which an unstable equilibrium point is between two stable equilibrium points. Two families of limit cycles bifurcating from the two unstable Hopf points intersect on the bifurcation curve (line) of the system. The pitchfork bifurcation point forks a quadratic curve with a negative slope which passes through the two 


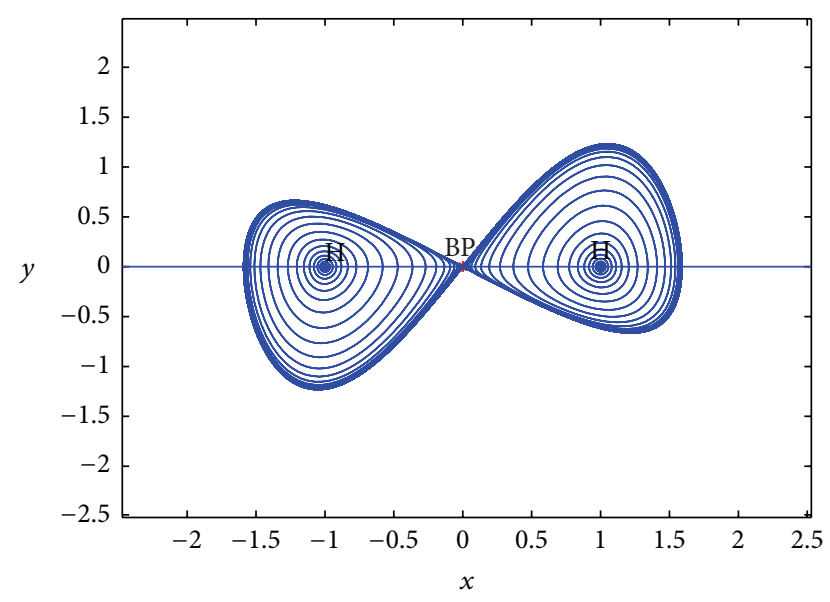

Figure 6: Phase plane of the DVP system (20) when $\beta=\alpha=$ $\alpha_{2}=1$ and $\alpha_{0}$ is varied where a pair of subcritical Hopf bifurcation points with families of limit cycles and a pitchfork bifurcation point (indicated by BP) are observed.

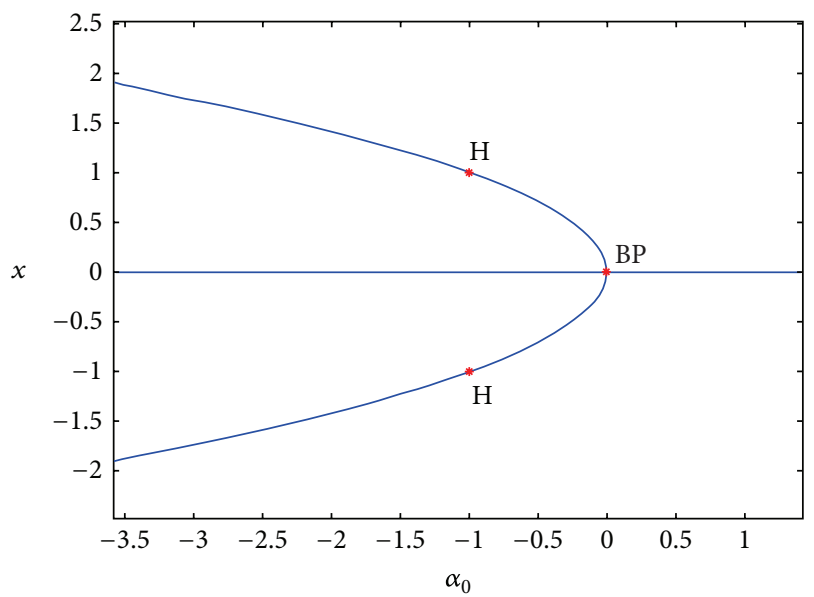

Figure 7: Bifurcation graph of the DVP system (20) in $x$ as $\alpha_{0}$ is varied.

subcritical Hopf bifurcation points so that the projection of the Lorenz attractor created would seem to intersect at the pitchfork bifurcation point BP in the two-dimensional $(x, y)$ graph. In this case, the equilibrium points of the system are again $(-1,0),(0,0)$, and $(1,0)$ and the eigenvalues are $\{ \pm i \sqrt{2}\},\{(1 \pm \sqrt{5}) / 2\}$, and $\{ \pm i \sqrt{2}\}$, respectively. The limit cycles go around two subcritical Hopf bifurcation points indicated by two positive first Lyapunov coefficients. The bifurcation analysis when $\alpha$ is varied shows a pitchfork bifurcation curve with numerous limit point bifurcation points on it. To understand the bifurcation properties in system (20), we calculate the averaged equations by the usual methods $[2,5,7]$

$$
\begin{gathered}
\dot{r}=\frac{\epsilon \beta}{2} r\left(1-\frac{r^{2}}{4}\right), \\
\dot{\theta}=\frac{1-\epsilon \alpha \alpha_{0}}{2}-\frac{3}{8}\left(\epsilon \alpha \alpha_{2} r^{2}\right)
\end{gathered}
$$

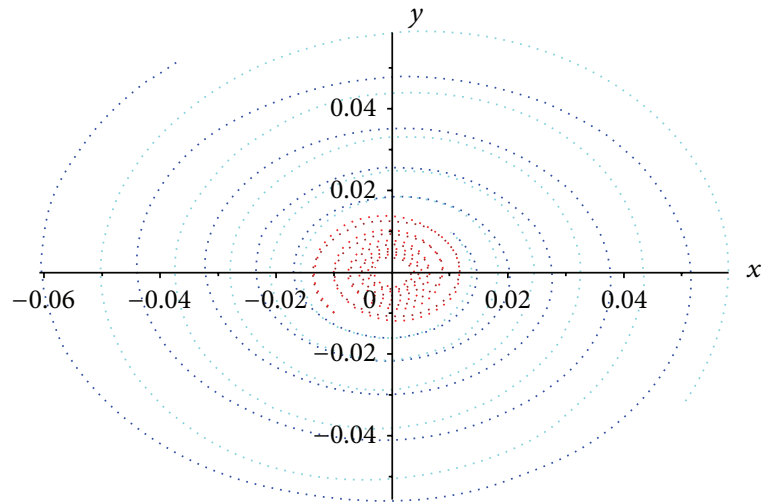

Averaged solutions $\beta>0$

Numerical solutions $\beta>0$ Averaged solutions $\beta<0 \quad \ldots$... Numerical solutions $\beta<0$

FIGURE 8: Numerical and averaged solutions of system (20) when $\alpha=6, \alpha_{0}=2, \alpha_{2}=2, \beta=-1$ and $\alpha=-6, \alpha_{0}=-2, \alpha_{2}=-2, \beta=1$ for $\epsilon=0.1$ near the origin where Hopf bifurcation is observed.

by introducing a small parameter $\epsilon, 0<\epsilon \ll 1$ and replacing $\alpha_{0}, \beta$ by $\epsilon \alpha_{0}, \epsilon \beta$, respectively.

Numerical and averaged solutions are given in Figure 8 where the origin is a stable (unstable) equilibrium point for $\beta<0, \alpha>0, \alpha_{0}>0, \alpha_{2}>0\left(\beta>0, \alpha<0, \alpha_{0}<0, \alpha_{2}<0\right)$.

3.1. The Maxwell-Bloch System. It has been of considerable interest to relate models for laser dynamics to VP and DVP systems. DVP model is known to give oscillatory solutions which includes several Hopf bifurcations [20]. The MaxwellBloch equations (to be referred to as MB henceforth) are

$$
\begin{gathered}
\dot{E}=-k E+g P, \\
\dot{P}=-\gamma_{\perp} P+g E \Delta, \\
\dot{\Delta}=-\gamma_{\|}\left(\Delta-\Delta_{0}\right)-4 g P E
\end{gathered}
$$

that contain one unstable equilibrium point at $E=P=0$, $\Delta=\Delta_{0}$ with one positive and two negative eigenvalues and pair of equilibrium points at either side with eigenvalues $\{\alpha \pm \beta i, \mu\}$. When $k=\sigma, \gamma_{\perp}=g^{2} / k=1, g^{2}\left(\Delta_{0} / k\right)=r$, and $\gamma_{\|}=b, \mathrm{MB}$ system (24) can be transformed into the Lorenz system about the equilibrium point $\Delta=\Delta_{0}$ by setting $x=E$, $y=g p / k, z=\Delta_{0}-\Delta[20]$. It was reported that operating point shows chaotic behavior that is similar to that of the Lorenz system [20]. The behavior of the MB system around the point $(0,0)$ is compared to the behavior of the DVP system around each of the equilibrium points $(0,0),(-1,0)$, and $(1,0)$ in Figure 9. For both systems, Figure 9 shows trajectories involving similar structures. 


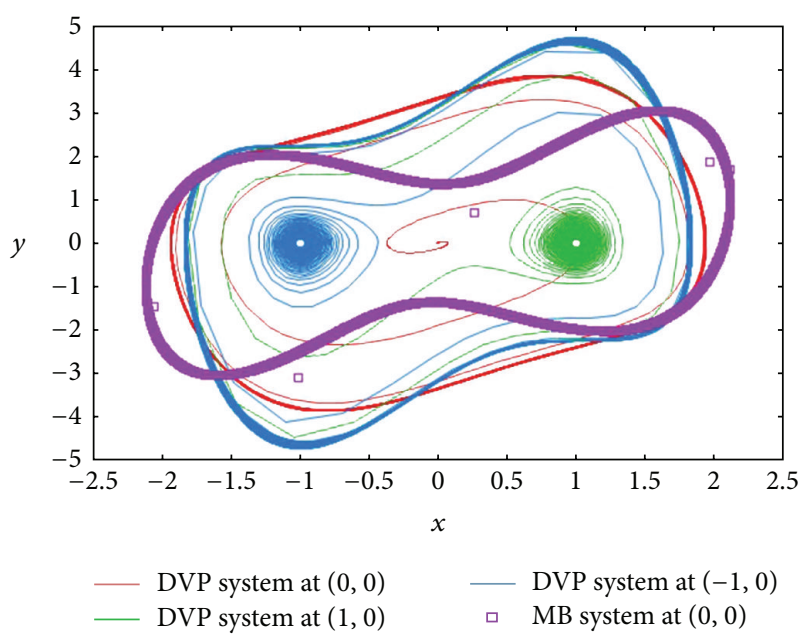

FIGURE 9: $x-y$ axis projection of the Maxwell-Bloch (MB) system $\left(k=11.75, g=25.18, \gamma_{\perp}=2.66, \gamma_{\|}=2.75, \Delta_{0}=28\right)$ and DuffingVan der Pol system $\left(\alpha=2, \alpha_{2}=2, \beta=2, \alpha_{0}=-2\right)$.

\section{The Generalized Van der Pol System including Quartic Self Nonlinearity}

Now we introduce a further generalized system including a quartic term in the parentheses. The generalization with this additional quartic term is

$$
\begin{gathered}
\dot{x}=\kappa y, \\
\dot{y}=-\alpha x\left(\alpha_{0}+\alpha_{2} x^{2}+\alpha_{4} x^{4}\right)+\beta y\left(1-x^{2}\right) .
\end{gathered}
$$

The averaged version of system (25) is

$$
\begin{gathered}
\dot{r}=\frac{\beta}{2} r\left(1-\frac{r^{2}}{4}\right), \\
\dot{\theta}=1-\frac{\kappa+\alpha \alpha_{0} \epsilon}{2}-\frac{\alpha r^{2}}{8}\left(3 \alpha_{2}+\frac{5 \alpha_{4}}{2} r^{2}\right),
\end{gathered}
$$

by introducing a small parameter $\epsilon, 0<\epsilon \ll 1$ and by replacing $\alpha_{0}, \beta$ by $\epsilon \alpha_{0}, \epsilon \beta$ in that given order.

According to the sign of $\Delta=\alpha^{2}-4 \beta$, the system exhibits different bifurcation scenarios where some of them are of interest and others are trivial.

A bifurcation analysis selecting $\kappa$ as the bifurcation parameter gives a pitchfork bifurcation point which forks a curve with numerous limit point bifurcation points as in the case for the DVP system. Selecting $\alpha$ as the bifurcation parameter, a pitchfork bifurcation is observed, and selecting $\beta$ as the bifurcation parameter, the usual subcritical Hopf bifurcation point scenario is observed. Other parameters do not give any bifurcation in this generalization.
The averaged version of the following further generalized system:

$$
\begin{gathered}
\dot{x}=\kappa y, \\
\dot{y}=-\alpha x\left(1+\alpha_{2} x^{2}+\alpha_{4} x^{4}+\alpha_{6} x^{6}+\alpha_{8} x^{8}\right) \\
+\beta y\left(1+\beta_{2} x^{2}+\beta_{4} x^{4}+\beta_{6} x^{6}+\beta_{8} x^{8}\right),
\end{gathered}
$$

is

$$
\begin{gathered}
\dot{r}=\frac{\kappa}{2} r\left(1+\frac{\beta_{1}}{4} r^{2}+\frac{\beta_{2}}{8} r^{4}+\frac{5 \beta_{3}}{64} r^{6}+\frac{7 \beta_{4}}{128} r^{8}\right), \\
\dot{\theta}=\frac{a+b}{2}-1+b\left(\frac{3 \alpha_{1}}{8} r^{2}+\frac{5 \alpha_{2}}{16} r^{4}+\frac{35 \alpha_{3}}{128} r^{6}+\frac{63 \alpha_{4}}{256} r^{8}\right),
\end{gathered}
$$

where $\alpha_{i}, \beta_{i} \in \mathbb{R},\{i=1, \ldots, 4\}, i \in \mathbb{Z}^{+}$, and scenarios depend on the characteristic of the numerous roots of $\dot{r}=0$.

\section{Conclusion}

The subcritical Hopf bifurcation point of the VP system at the origin is inherited through all polynomial generalizations in the VP system. Generally a pitchfork bifurcation is observed in all bifurcation graphs which forks into higher bifurcations. Many limit point bifurcations are observed for all generalizations. Pitchfork and limit point bifurcations are observed for generalizations of VP system including quadratic selfcoupling. Hopf bifurcation has also been studied using the normal form method as given in Definition 2, for the eigenvalues of the linearized system at equilibrium points. For generalized VP systems considered in this study, averaging over the period of the linear part, a limit cycle property is observed for all possible cases. To this approximation, a change in the nonlinearity of the system does not change the occurrence of limit cycle phenomena. However $\dot{\theta}$ in polar coordinates does not remain constant. Also the case where the bifurcation curves cycle and oscillate around a pitchfork bifurcation point between two Hopf points only occurs for the original DVP system which is nonintegrable. A relation between the DVP and MB systems where both exhibit Lorenz-like behavior has been shown.

\section{Conflict of Interests}

The authors declare that there is no conflict of interests regarding the publication of this paper.

\section{Acknowledgments}

The authors would also like to thank the editor and referees for their valuable and constructive comments. The authors would like to thank Professor Dr. Ferdinand Verhulst for his valuable suggestions and comments that helped to improve the paper throughout the process. 


\section{References}

[1] B. van der Pol, "On relaxation-oscillations," The London and Edinburgh Philosophical Magazine and Journal of Science, vol. 7, pp. 978-992, 1927.

[2] J. Guckenheimer and P. Holmes, Nonlinear Oscillations, Dynamical Systems, and Bifurcations of Vector Fields, Springer, New York, NY, USA, 2002.

[3] R. H. Rand and D. Armbruster, Perturbation Methods, Bifurcation Theory and Computer Algebra, vol. 65 of Applied Mathematical Sciences, Springer, New York, NY, USA, 1987.

[4] S. H. Strogatz, Nonlinear Dynamics and Chaos: With Applications to Physics, Biology, Chemistry and Engineering, Westview Press, 2001.

[5] F. Verhulst, Nonlinear Differential Equations and Dynamical Systems, Springer, New York, NY, USA, 2nd edition, 2006.

[6] F. Veerman and F. Verhulst, "Quasiperiodic phenomena in the Van der Pol-Mathieu equation," Journal of Sound and Vibration, vol. 326, no. 1-2, pp. 314-320, 2009.

[7] J. A. Sanders, F. Verhulst, and J. Murdock, Averaging Methods in Nonlinear Dynamical Systems, vol. 59 of Applied Mathematical Sciences, Springer, New York, NY, USA, 2007.

[8] Y. A. Kuznetsov, Elements of Applied Bifurcation Theory, vol. 112 of Applied Mathematical Sciences, Springer, New York, NY, USA, 1995.

[9] D. W. Jordan and P. Smith, Nonlinear Ordinary Differential Equations: An Introduction to Dynamical Systems, vol. 2 of Oxford Texts in Applied and Engineering Mathematics, Oxford University Press, Oxford, UK, 1999.

[10] J. K. Hale and H. Koçak, Dynamics and Bifurcations, vol. 3 of Texts in Applied Mathematics, Springer, New York, NY, USA, 1991.

[11] M. Xiao and J. Cao, "Genetic oscillation deduced from Hopf bifurcation in a genetic regulatory network with delays," Mathematical Biosciences, vol. 215, no. 1, pp. 55-63, 2008.

[12] J. Cao and M. Xiao, "Stability and Hopf bifurcation in a simplified BAM neural network with two time delays," IEEE Transactions on Neural Networks, vol. 18, no. 2, pp. 416-430, 2007.

[13] M. Xiao and J. Cao, "Delayed feedback-based bifurcation control in an Internet congestion model," Journal of Mathematical Analysis and Applications, vol. 332, no. 2, pp. 1010-1027, 2007.

[14] B. J. W. S. Rayleigh, The Theory of Sound, Dover, New York, NY, USA, 1945.

[15] A. Liénard, "Etude des oscillations entretenues," Revue Générale de l'Électricité, vol. 23, pp. 901-912, 946-954, 1928.

[16] F. C. Moon and P. J. Holmes, "A magnetoelastic strange attractor," Journal of Sound and Vibration, vol. 65, no. 2, pp. 275296, 1979.

[17] W. Yu and J. Cao, "Hopf bifurcation and stability of periodic solutions for van der Pol equation with time delay," Nonlinear Analysis: Theory, Methods \& Applications, vol. 62, no. 1, pp. 141165, 2005.

[18] A. Doelman and F. Verhulst, "Bifurcations of strongly nonlinear self-excited oscillations," Mathematical Methods in the Applied Sciences, vol. 17, no. 3, pp. 189-207, 1994.

[19] I. Kusbeyzi, O. O. Aybar, and A. Hacinliyan, "Stability and bifurcation in two species predator-prey models," Nonlinear Analysis: Real World Applications, vol. 12, no. 1, pp. 377-387, 2011.

[20] I. Kusbeyzi, O. O. Aybar, and A. S. Hacinliyan, "Approximate solutions of Maxwell Bloch equations and possible Lotka
Volterra type behavior," Nonlinear Dynamics, vol. 62, no. 1-2, pp. 17-26, 2010. 


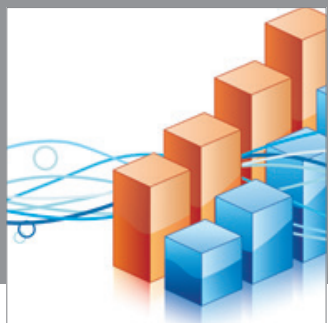

Advances in

Operations Research

mansans

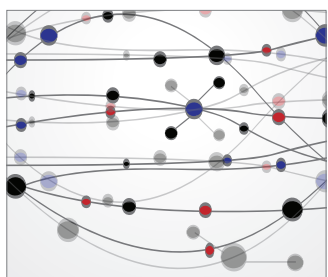

The Scientific World Journal
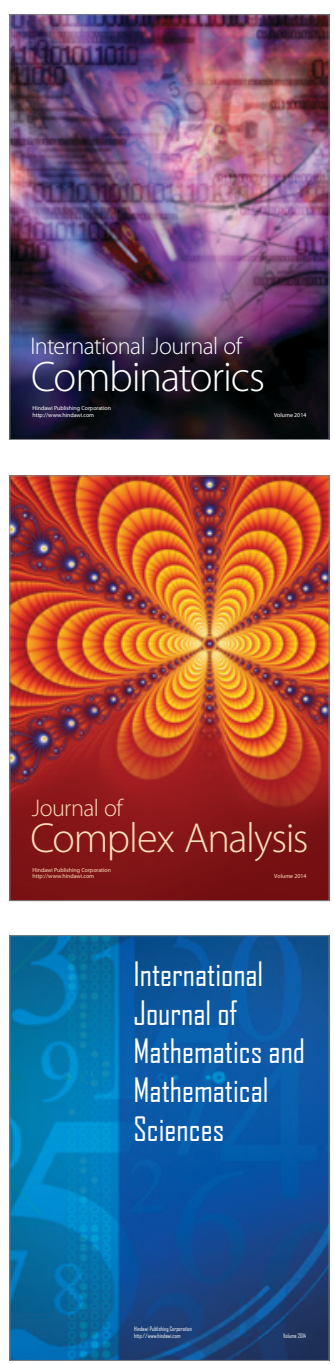
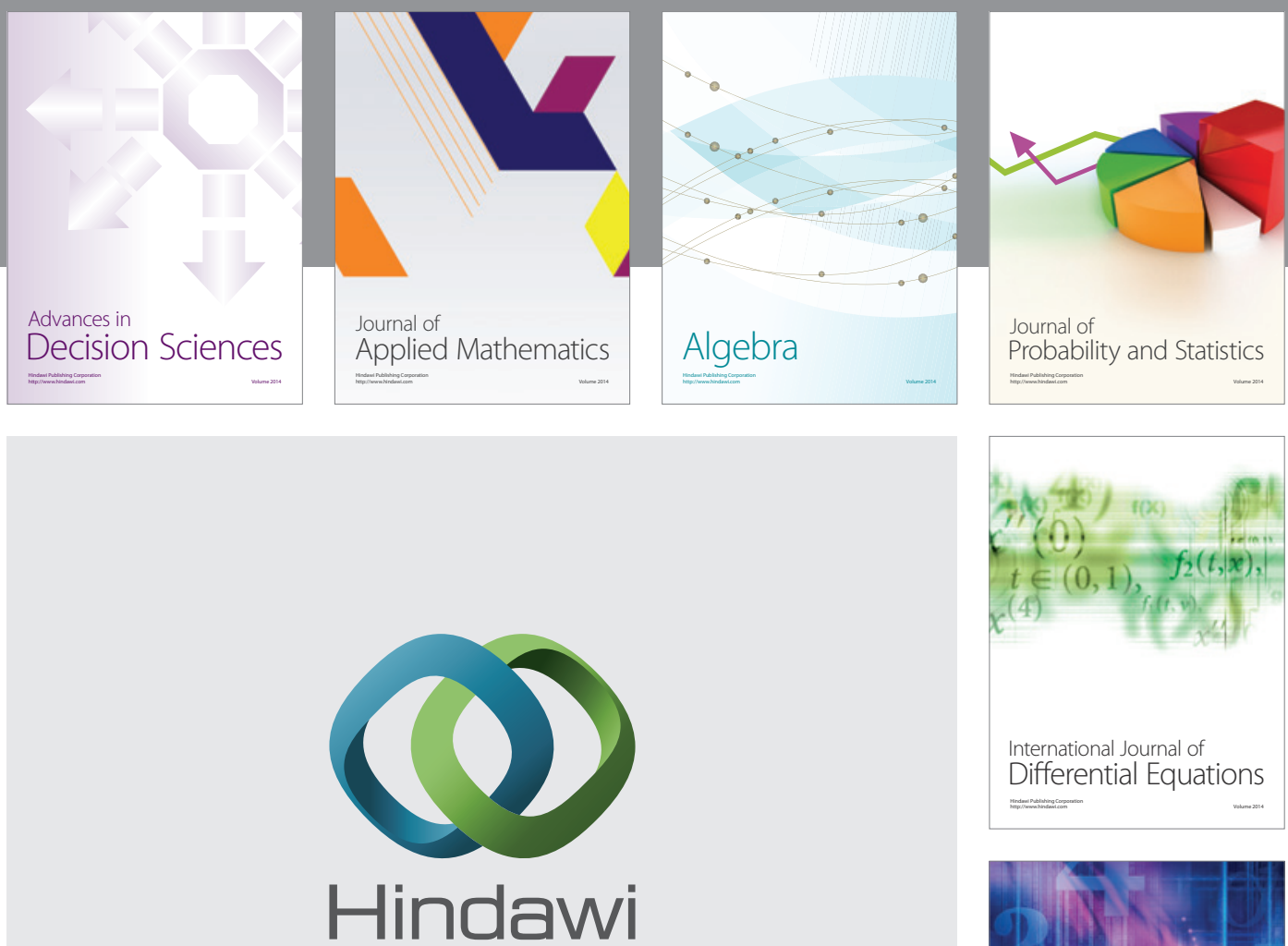

Submit your manuscripts at http://www.hindawi.com
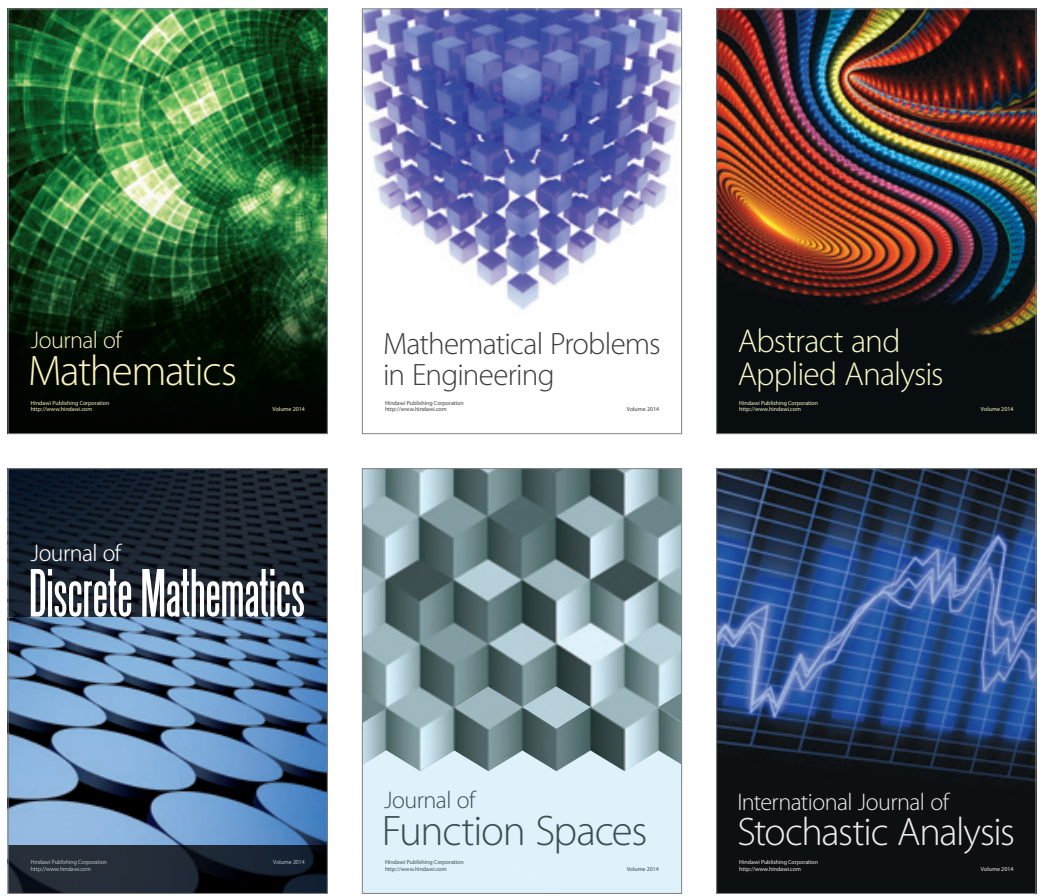

Journal of

Function Spaces

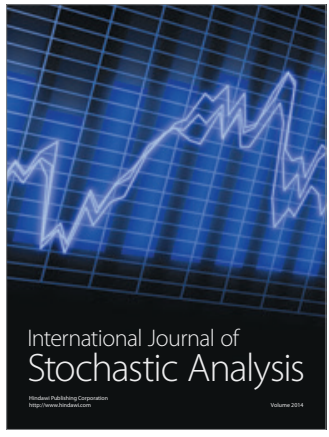

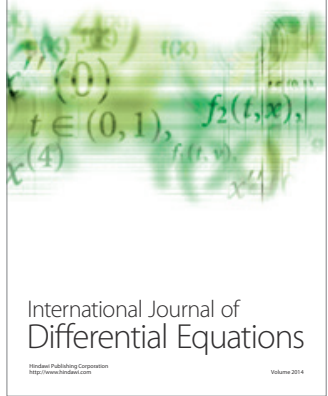
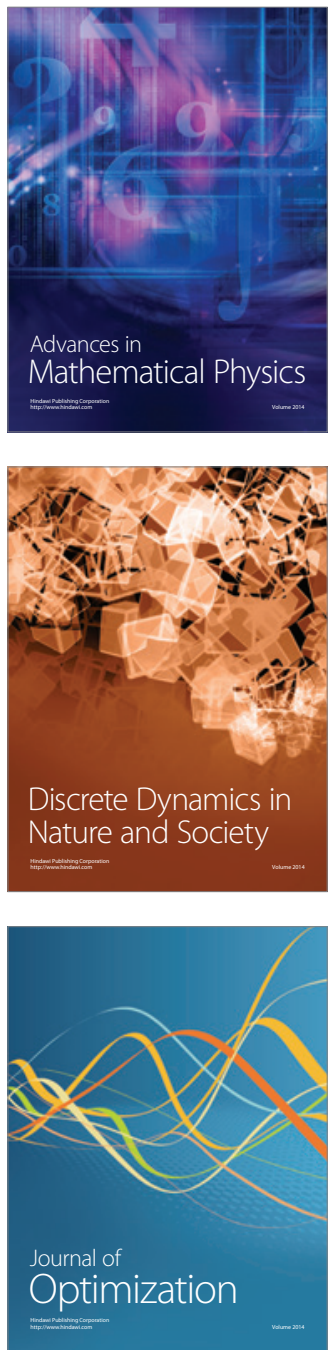